\title{
EXPLORANDO LA INTELIGENCIA EMOCIONAL PERCIBIDA EN TRES FRANJAS ETARIAS UN ESTUDIO REALIZADO EN LA ARGENTINA
}

Claudia Josefina Arias ${ }^{1}$

Maria Florencia Giuliani²

resumen

La inteligencia emocional percibida consiste en un conjunto de habilidades de procesamiento emocional. Incluye la capacidad para percibir los propios estados emocionales - Atención emocional -, comprenderlos y expresarlos lingüísticamente - Claridad de sentimientos - y regularlos adaptativamente - Reparación emocional. Este constructo se encuadra dentro del enfoque de la Psicología Positiva, ha sido asociado a la resiliencia y funciona como amortiguador de las respuestas de estrés y como un factor protector contra el burnout. Este estudio explora y compara las características

1 Licenciada en Psicología, Magíster en Psicología Social. Docente de grado y posgrado e investigadora. Facultad de Psicología. Universidad Nacional de Mar del Plata. Directora del proyecto Exploración de las Relaciones entre dimensiones de las emociones percibidas y grupo de edad en personas de Mar del Plata y Buenos Aires. E-mail: cjarias@mdp.edu.ar

2 Licenciada en Psicología. Becaria de Investigación de la Universidad Nacional de Mar del Plata, categoría Iniciación. Este trabajo se realizó con los fondos de dicha beca, dentro del marco del grupo de investigación "Evaluación Psicológica", en el proyecto "Exploración de las Relaciones entre dimensiones de las emociones percibidas y grupo de edad en personas de Mar del Plata y Buenos Aires". E-mail: mariaflorenciagiuliani@gmail.com 
de la inteligencia emocional percibida en personas de tres grupos de edad: 1) 15 a 24; 2) 30 a 45 y 3) 60 a 75 años, mediante un diseño transversal, descriptivo-correlacional. Se realizó un muestreo no probabilístico-intencional de 252 sujetos de ambos sexos a los que se administró la Escala de Metaconocimiento sobre Estados Emocionales. Los resultados señalan que los participantes de 60 a 75 años presentan mayor capacidad de comprensión y expresión emocional que los de los grupos restantes, así como mayor capacidad de regular sus emociones que los del grupo de 15 a 24 años. Estos resultados apoyan los antecedentes que señalan que los adultos mayores presentan un perfil de mayor desarrollo de las habilidades de Inteligencia Emocional que las personas de menor edad. Esto implica que los adultos mayores tienen disponibles estos efectivos recursos emocionales para afrontar y adaptarse a los desafíos de la vida cotidiana en la vejez.

palabras clave Inteligencia Emocional. Grupo de Edad. Envejecimiento Positivo.

\section{Introducción}

Dentro del estudio de las emociones y el procesamiento emocional, se ha configurado un campo de investigaciones que aborda el estudio de las habilidades para modificar los propios estados afectivos. La modificación de la propia experiencia emocional se produce cuando la reacción afectiva automática, influenciada por la calidad del estímulo, las características de personalidad y la situación contextual, no favorece la consecución de las metas deseadas. En tal situación, se vuelve necesario desplegar recursos y habilidades personales con el fin de cambiar esa respuesta (KOOLE; VAN DER LEEN; SHEPPES, 2011). Estas destrezas sirven para amortiguar las respuestas de estrés producidas por los problemas de la vida cotidiana (BLANCHARD-FIELDS, 2007) y funcionan como factores protectores contra el burnout (EXTREMERA; DURÁN; REY, 2007) e ideas intrusivas en situaciones de estrés agudo (RAMOS-DÍAZ; FERNÁNDEZ-BERROCAL; EXTREMERA, 2007).

Para este estudio resultan de especial interés las habilidades de comprensión y regulación emocional metacognitivas. En este nivel del procesamiento emocional es posible registrar explícitamente las emociones experimentadas, 
monitoreando y detectando desvíos del estado de ánimo basal. Este proceso de control de la propia experiencia facilita las respuestas de afrontamiento y reparación afectiva (BRIÑOL; PETTY; RUCKER, 2006).

El procesamiento de las emociones mediado por procesos metacognitivos es explorado a través del constructo "Inteligencia Emocional Percibida" (IEP), propuesto por Mayer y Stevens (1994). Puntualmente, la inteligencia emocional consiste en un conjunto de habilidades que facilita la resolución de problemas de la vida cotidiana, mediante la utilización de conocimiento metacognitivo acerca del funcionamiento de las emociones a nivel intra e interpersonal (MAYER; SALOVEY, 1997). Se la considera una inteligencia en tanto se trata de una habilidad mental y no de estilos conductuales; correlaciona con otros tipos de inteligencia pero no se solapa con ellas y se desarrolla con la edad y la experiencia (MAYER; CARUSO; SALOVEY, 2000).

La inteligencia emocional percibida comprende tres dominios: 1) Atención Emocional, 2) Claridad Emocional y 3) Reparación Emocional. El primero se define como la predisposición de los individuos a estar pendientes y a registrar sus emociones y estados de ánimo. Incluye la capacidad de atender a la expresión verbal de las emociones, las configuraciones faciales, los movimientos corporales y el tono de voz, así como la habilidad para discernir entre las expresiones sinceras y fingidas. Este dominio es el más básico, sin un buen desarrollo de éste resulta poco probable que las otras ramas tengan niveles de funcionamiento adecuados. La capacidad para reconocer emociones es fundamental para la interacción humana y la conducta prosocial, así como para el aprendizaje social. Se desarrolla al momento del nacimiento y dentro del primer año de vida, reflejando la emergencia de circuitos neuronales precableados. Para lograr la maduración de la habilidad de reconocimiento emocional se necesita de una mínima provisión ambiental que estabilice ciertas conexiones y produzca el refinamiento de la red funcional. La adquisición temprana de esta habilidad produce que los niños pequeños atiendan preferencialmente a expresiones emocionales, facilitando el aprendizaje de representaciones de patrones expresivos característicos de la especie (LEPPÄNEN, 2011).

El segundo dominio, la Claridad de Sentimientos, refiere a la capacidad de comprender los estados emocionales: desglosar las respuestas emocionales complejas y multimodales - para poder reconocer sus componentes y categorizarlas -, entender sus posibles combinaciones, dinámica y transiciones entre diferentes emociones y la aparición simultánea de afectos contradictorios. Es una habilidad que puede utilizarse para anticipar y para comprender retrospectivamente las causas de las reacciones emocionales y 
sus posibles consecuencias. También incluye a la posibilidad de referirse a ellas lingüísticamente (MAYER; CARUSO; SALOVEY, 2000). La Claridad de Sentimientos es considerada una estrategia preventiva de regulación de los estados emocionales, en tanto el acto de interpretar y otorgar sentido a la experiencia emocional favorece que la misma no sea sentida como disruptiva (EXTREMERA; FERNÁNDEZ-BERROCAL, 2005). En tercer lugar, la Reparación Emocional refiere a las habilidades de regular las emociones adaptativamente, decidir si se las considerará relevantes o no en alguna situación específica. Esta capacidad implica poder incrementar o disminuir tanto las emociones positivas como las negativas. El manejo de las emociones involucra el monitoreo y distinción efectiva de las mismas, la motivación para regularlas y la percepción de autoeficacia en esa tarea, así como la posibilidad de intervenir sobre ellas y modificarlas efectivamente (MAYER; CARUSO; SALOVEY, 2000). La Reparación Emocional ha sido considerada una habilidad paliativa, en tanto se despliega cuando los estados emocionales no deseados o incompatibles con las metas son registrados conscientemente (EXTREMERA; FERNÁNDEZ-BERROCAL, 2005).

Según Thayer et al. (2003), las personas con altos niveles de Atención Emocional y similares habilidades de Claridad y Reparación presentan los efectos positivos de un mejor procesamiento emocional - identificando expresiones faciales con mayor exactitud, mayor complejidad emocional y mayor sensibilidad -, mientras que elevados niveles de Atención pero no de los restantes dos dominios, se asocia con rumiación y síntomas depresivos. Estos dos tipos de configuraciones pueden ser pensados como perfiles de habilidades de IEP más y menos saludables, respectivamente. En relación a la Inteligencia Emocional, se han investigado diversos factores moduladores, entre ellos, la edad (LAWTON et al., 1992; KAFETSIOS, 2004; EXTREMERA; FERNÁNDEZ-BERROCAL; SALOVEY, 2006), la teoría de la mente (DUVAL et al., 2011) y los estereotipos de género y las pautas culturales (MAUSS; BUNGE; GROSS, 2007). El presente estudio se centra en la edad como variable moduladora de los niveles de IEP. Con el fin de comprender más profundamente la forma en que incide este factor en la Atención Emocional, la Claridad de Sentimientos y la Reparación Emocional, presentamos a continuación una serie de teorías desarrolladas específicamente para la explicación de las características de las habilidades emocionales en el transcurso vital y la vejez.

En primer lugar, la propuesta por Labouvie-Vief (LABOUVIE-VIEF et al., 2007) señala que los adultos mayores presentan mayor optimización emocional - más emociones positivas que negativas - que los jóvenes y los 
adultos de mediana edad, aunque éstas son de menor complejidad. Según los autores, esto implica que los adultos mayores procesan sus emociones de una manera simplificada, dicotómica y estereotipada en relación a la variabilidad que caracteriza al pensamiento emocional de los adultos jóvenes. Estas características se deberían al deterioro normal de las funciones ejecutivas que se produce a partir de los 60 años. Dicho deterioro está moderado por la clase social, la educación y el perfil de salud física y mental. Asimismo, las diferencias entre los grupos de edad desaparecen cuando se evalúan las emociones referidas a problemáticas que involucran la seguridad, la moral y la verdad o se produce una identificación importante con metas personales. Es decir que a pesar de haber hallado evidencia de un deterioro normativo en lo que hace a la compresión de las emociones, el desempeño estaría influido por otros factores.

En segundo término, la teoría de la selectividad socioemocional (CHARLES; CARSTENSEN, 2007; CARSTENSEN; MIKELS; MATHER, 2006) explora los factores motivacionales, extrínsecos al deterioro cognitivo, que afectan al procesamiento emocional. Esta teoría propone la existencia de un cambio normativo en las metas motivacionales a lo largo de la adultez. Este cambio se debe a que se percibe el tiempo vital restante como acotado, finito, en contraste al carácter ilimitado que presenta en la juventud. La nueva concepción produce que se prioricen metas relacionadas con el estado de ánimo y la búsqueda de significado, en lugar de la acumulación de información para la obtención de ventajas futuras, característica de la juventud. Este cambio motivacional repercute en el desarrollo de lo que los autores han llamado "efecto de positividad" (CARSTENSEN; MIKELS, 2005). El mismo consiste en un patrón normativo de selección de la información ambiental: en la juventud se prefiere el material emocionalmente negativo, porque favorece la adaptación al medio, mientras que a lo largo de la adultez el sesgo se transforma en una preferencia desproporcionada por la información positiva. Esta nueva dirección en la selección de la información impacta en una profundización de los significados personales asociados a las emociones y al bienestar y una mayor implicación en actividades que produzcan satisfacción personal. Al contrario de lo planteado por Labouvie-Vief (LABOUVIE-VIEF et al., 2007), esta línea propone que el incremento de la edad repercute positivamente en la capacidad de distinguir las emociones, comprenderlas con mayor riqueza y complejidad así como de optimizar las habilidades de regulación emocional, debido a los cambios ya referidos.

Por otra parte, Blanchard-Fields (2007) presenta un enfoque del procesamiento emocional centrado en el estudio de problemas de la vida cotidiana, 
desestructurados y dinámicos, en los que se requieren habilidades y conocimientos prácticos para aprovechar las oportunidades y responder a las demandas del ambiente. La investigadora afirma que los recursos que las personas ponen en juego a la hora de resolver estas situaciones emocionalmente problemáticas se mantienen y optimizan con la edad. En sus investigaciones estableció que los adultos mayores comprenden y regulan sus emociones de manera cualitativamente distinta de los adultos jóvenes: los primeros presentan respuestas más flexibles, complejas - heterogéneas -, maduras y efectivas que los últimos (BLANCHARD-FIELDS; STEIN; WATSON, 2004). Asimismo, en situaciones de problemas con vínculos cercanos, los adultos mayores prefieren la utilización de estrategias de regulación emocional focalizadas en sí mismos, como la reflexión y reevaluación de situaciones. La autora propone que los adultos mayores, además de los cambios en las estrategias de selección (CHARLES; CARSTENSEN, 2007; SCHEIBE; CARSTENSEN, 2010) y de la compensación ante el deterioro de funciones cognitivas (LABOUVIE-VIEF et al., 2007), presentan mayor competencia para regular sus emociones en tanto han aprendido y practicado estas habilidades a lo largo de su vida, convirtiéndose en expertos en esta tarea.

Los antecedentes empíricos dan cuenta de trayectorias evolutivas diferenciales para las habilidades de procesamiento emocional que incluyen la inteligencia emocional percibida. Mientras que la Atención emocional se desarrolla en los primeros meses de vida y se mantiene estable a través de los años, la Claridad y la Reparación se desarrollan y modifican hasta las últimas etapas de la vida, dependiendo en mayor medida de la experiencia para adquirirse y optimizarse (LEPPÄNEN, 2011).

En lo referido a la Atención, Lobue y De Loache (2010) hallaron que los niños de 6 a 7 meses diferencian entre las expresiones faciales de temor y enojo. Esta capacidad se mantiene relativamente estable a través de la vida. Específicamente en estudios sobre el reconocimiento de expresiones faciales del miedo, los niños presentan desempeños similares a los adultos (PASCALIS et al., 2005).

En cuanto a la relación entre la comprensión y regulación de las emociones y el envejecimiento, los antecedentes muestran algunas discrepancias. En lo que respecta al control de las propias emociones, Lawton et al. (1992), en un estudio pionero sobre el tema, encontraron que los adultos mayores presentan mayor control emocional, estabilidad en el estado de ánimo, moderación de los afectos, nivelación de los estados de ánimo positivos y menor surgencia - dominancia y competitividad -, responsividad psicofisiológica y búsqueda de sensaciones que los más jóvenes. Gross et al. 
(1997) hallaron que el control emocional aumentaba con la edad. Por otra parte, Nashiro, Sakaki y Mather (2011) mostraron que en tareas de regulación emocional, los adultos mayores presentan mayor actividad en la corteza cerebral prefrontal que los adultos jóvenes, es decir mayor control cognitivo sobre la experiencia emocional.

También en un estudio comparativo, Kafetsios (2004) y Extremera, Fernández-Berrocal y Salovey (2006) hallaron puntajes más altos en comprensión, facilitación y manejo de las emociones en el grupo de más edad. Según Shiota y Levenson (2009), los adultos mayores, en comparación con los adultos jóvenes, implementan menor reevaluación cognitiva desapegada - focalizada en aspectos racionales -, mayor reevaluación cognitiva positiva - atención en aspectos y consecuencias negativas y positivas - y similar capacidad de supresión conductual; por lo que los primeros parecen tener una compresión más compleja de las situaciones conflictivas que los últimos. En un estudio longitudinal, Carstensen et al. (2011) hallaron que el envejecimiento estaba asociado positivamente con el bienestar emocional, la estabilidad emocional y la complejidad afectiva. Por su parte, Hay y Diehl (2011), en un estudio realizado con sujetos de 18 a 89 años, hallaron que los adultos mayores no presentaban diferencias significativas respecto de los jóvenes en lo referido a complejidad afectiva - diferenciación emocional y co-ocurrencia en afecto positivo y negativo - y mostraban patrones más adaptativos de regulación emocional. Específicamente explorando la relación entre edad y uso de la compresión emocional para la regulación de los estados afectivos, Blanchard-Fields y Coats (2008) no hallaron asociación $(r=0.04)$, sugiriendo que la complejidad emocional no sería un factor determinante de la regulación emocional. Al contrario, Márquez González et al. (2008) encontraron mayores puntuaciones en estrategias más saludables de regulación emocional en las personas jóvenes. Asimismo, Mendes (2010) afirma que la información interoceptiva resulta crítica para comprender las propias emociones. La investigadora afirma que este tipo de registro decae en los adultos mayores, por lo que confían más en los estímulos externos para comprender sus estados emocionales y la comprensión de las emociones se ve deteriorada con el incremento de la edad.

En función de los desarrollos teóricos y hallazgos empíricos revisados, la presente investigación propone el estudio de la inteligencia emocional percibida, explorando y comparando sus características en tres grupos de edad. Se propone como hipótesis de trabajo que los adultos mayores presentarán niveles similares de atención emocional y mayores de claridad de sentimientos y reparación emocional que los restantes grupos de edad. 
Este trabajo fue realizado en el marco de una beca de investigación obtenida mediante concurso. El proyecto fue aprobado por una comisión evaluadora de investigadores expertos y fue subsidiado por la Secretaría de Ciencia y Técnica de la Universidad Nacional de Mar del Plata. El mismo se insertó en un proyecto de investigación mayor, denominado: "Exploración de las relaciones entre dimensiones de las emociones percibidas y grupo de edad en personas de Mar del Plata y Buenos Aires", dentro del grupo de investigación "Evaluación Psicológica", radicado en la Facultad de Psicología de la Universidad Nacional de Mar del Plata.

Durante el proceso de recolección de datos, las personas que formaron parte de la muestra firmaron un Consentimiento Informado. En el mismo se explicitaba que su participación era de carácter voluntario, anónimo, confidencial y que la información derivada de la investigación se utilizaría con fines exclusivamente científicos, bajo la jurisdicción de la Ley Nacional 25.326 de protección de los datos personales.

En cuanto a los aspectos metodológicos, el diseño de la investigación fue de tipo no experimental, transversal/correlacional. Se trabajó con una muestra intencional de personas residentes en la ciudad de Mar del Plata. La recolección de datos se realizó entre septiembre del 2010 y marzo del 2011. Los criterios de inclusión para formar parte de la muestra fueron cumplir con la edad requerida y no presentar dificultades para la comprensión del cuestionario. Se seleccionaron personas que fuesen autoválidas y que vivieran en hogares particulares. Los instrumentos fueron administrados por los investigadores en espacios de entrevistas individuales. Para establecer el contacto con las personas e invitarlas a participar, se pidió autorización a organizaciones que trabajaran con población general o grupos etarios específicos (por ejemplo, escuelas secundarias y clubes para personas de tercera edad). También se contactaron personas en lugares de esparcimiento público, como plazas y espacios peatonales.

La muestra quedó conformada por 252 sujetos, de los cuales 146 (57,9\%) fueron mujeres y $106(42,1 \%)$ varones. Se consideraron 3 grupos de edad: de 15 a 24 años (grupo 1), de 30 a 45 años (grupo 2) y de 60 a 75 años (grupo 3). Las medias de edad fueron: para el grupo 1 de 17,4 años (DS 2,9), para el grupo 2, de 36,5 años (DS 4,9) y para el grupo 3, de 67,5 (DS 5,3). En cuanto a la distribución del género según grupo de edad, los porcentajes de hombres y mujeres en los tres grupos fue similar (ver tabla 1), presentando la mayor diferencia el grupo 2, que estuvo compuesto por 51 mujeres y 33 hombres. En 
cuanto al nivel de escolarización (ver tabla 2), la mayoría de los sujetos pertenecientes al grupo $1(75,0 \%)$ presentaron nivel primario completo. En el caso del grupo 2, el 53,6\% había completado el nivel universitario y en el grupo 3, el $42,9 \%$ de los entrevistados había finalizado el nivel secundario. Respecto de las características del grupo de convivencia de los entrevistados (ver tabla 3), en el caso del grupo 1, la mayoría de los sujetos vivían con familiares, al igual que el porcentaje mayoritario del grupo 2. Respecto del grupo 3, el $34,5 \%$ de los entrevistados no convivía con otras personas.

Tabla 1 - Género de los participantes de la investigación según grupo de edad.

\begin{tabular}{l|c|c|c|c|c|c}
\hline \multirow{2}{*}{ Grupo de edad } & \multicolumn{2}{|c|}{ Mujeres } & \multicolumn{2}{c|}{ Varones } & \multicolumn{2}{c}{ Total } \\
\cline { 2 - 7 } & $\mathbf{n}$ & $\%$ & $\mathbf{N}$ & $\%$ & $\mathbf{n}$ & $\%$ \\
\hline 15 a 25 años & 45 & 53,6 & 39 & 46,4 & 84 & 100,0 \\
\hline 40 a 55 años & 51 & 60,7 & 33 & 39,3 & 84 & 100,0 \\
\hline 65 a 75 años & 50 & 59,5 & 34 & 40,5 & 84 & 100,0 \\
\hline Total & 146 & 57,9 & 106 & 42,1 & 252 & 100,0 \\
\hline
\end{tabular}

Tabla 2 - Nivel de escolarización máximo alcanzado por los participantes de la investigación según grupo de edad.

\begin{tabular}{l|c|c|c|c|c|c|c|c|c|c}
\hline \multirow{2}{*}{ Grupo de edad } & \multicolumn{2}{|c|}{ Sin estudios } & \multicolumn{2}{|c|}{ Primaria } & \multicolumn{2}{c|}{ Secundaria } & \multicolumn{2}{c|}{ Universitario } & \multicolumn{2}{c}{ Total } \\
\cline { 2 - 11 } & $\mathbf{n}$ & $\%$ & $\mathbf{n}$ & $\%$ & $\mathbf{n}$ & $\%$ & $\mathbf{n}$ & $\%$ & $\mathbf{n}$ & $\%$ \\
\hline 15 a 25 años & 0 & 0 & 63 & 75,0 & 21 & 25,0 & 0 & 0 & 84 & 100,0 \\
\hline 40 a 55 años & 0 & 0 & 4 & 4,8 & 35 & 41,7 & 45 & 53,6 & 84 & 100,0 \\
\hline 65 a 75 años & 1 & 1,2 & 24 & 28,6 & 36 & 42,9 & 23 & 27,4 & 84 & 100,0 \\
\hline
\end{tabular}

Tabla 3 - Grupo de convivencia de los participantes de la investigación según grupo de edad.

\begin{tabular}{l|c|c|c|c|c|c|c|c|c|c}
\hline \multirow{2}{*}{ Grupo de edad } & \multicolumn{2}{|c|}{ Solo } & \multicolumn{2}{c|}{ Familia } & \multicolumn{2}{c|}{ Pareja } & \multicolumn{2}{c|}{ Otros } & \multicolumn{2}{c}{ Total } \\
\cline { 2 - 12 } & $\mathbf{n}$ & $\%$ & $\mathbf{n}$ & $\%$ & $\mathbf{N}$ & $\%$ & $\mathbf{n}$ & $\%$ & $\mathbf{n}$ & $\%$ \\
\hline 15 a 25 años & 3 & 3,6 & 73 & 92,8 & 3 & 3,6 & 5 & 6,0 & 84 & 100,0 \\
\hline 40 a 55 años & 5 & 6,0 & 42 & 50,0 & 17 & 20,2 & 20 & 23,8 & 84 & 100,0 \\
\hline 65 a 75 años & 29 & 34,5 & 8 & 9,5 & 27 & 32,1 & 20 & 23,8 & 84 & 100,0 \\
\hline
\end{tabular}


En el trabajo de campo se implementaron los siguientes instrumentos de recolección de datos:

- Cuestionario de datos sociodemográficos. Se exploró edad, sexo, grupo conviviente y nivel de instrucción a fin de caracterizar la muestra.

- Escala Rasgo de Metaconocimientos sobre Estados Emocionales (FERNÁNDEZ-BERROCAL; EXTREMERA; RAMOS, 2004). Este instrumento evalúa los niveles de inteligencia emocional intrapersonal percibida, explorando tres factores: 1) atención emocional, 2) claridad de sentimientos y 3) reparación emocional. El primero refiere a la tendencia a registrar y pensar acerca de los estados anímicos (p.e. el ítem 3 "Normalmente dedico tiempo a pensar en mis emociones"). El segundo, Claridad de sentimientos, evalúa la comprensión de los propios estados emocionales (p.e. el ítem 13 "A menudo me doy cuenta de mis sentimientos en diferentes situaciones"). Por último, Reparación emocional explora las habilidades de regulación emocional intrapersonales (p.e. el ítem 21 "Si doy demasiadas vueltas a las cosas, complicándolas, trato de calmarme"). Si bien la escala no provee de un puntaje total, es posible evaluar globalmente el constructo a partir de la interpretación de los puntajes de las tres dimensiones en términos de un perfil de inteligencia emocional (THAYER et al., 2003). El formato de la escala es de tipo Likert, consta de 24 ítems con 5 opciones de respuesta según grado de acuerdo (1: nada de acuerdo a 5: totalmente de acuerdo). Las dimensiones poseen ocho ítems cada una, pudiendo obtener una puntuación mínima de 8 y máxima de 40. Regner (2009) investigó las propiedades psicométricas de la prueba en población argentina. Respecto del índice de consistencia interna, Salguero et al. (2010) encontraron que la escala tenía muy buenas propiedades psicométricas (Atención emocional: $\alpha=.90$, para Claridad de sentimientos, $\alpha=.90$ y para Reparación emocional $\alpha=.86$ ). A partir del análisis de los datos de la presente investigación, se encontraron valores similares a los anteriores: Atención emocional $\alpha=.86$, Claridad de sentimientos $\alpha=.85$ y Reparación emocional $\alpha=.83$.

\section{Análisis de datos}

Se efectuó un análisis estadístico de los datos mediante el uso del paquete informatizado InfoStat. Luego de comprobar que se cumplieran los supuestos para su aplicación, se utilizó el Análisis de Varianza (ANOVA) a fin de identificar si existían diferencias significativas (con un $\alpha$ de .05) entre 
las medias de los grupos de edad en cada dimensión de la IEP. En los casos en los que se identificó la existencia de diferencias significativas, se aplicó como prueba de contraste a posteriori y con el objeto de comparar los pares de medias simples el Test de Comparación múltiple de Mínima Diferencia Significativa, también considerando un nivel $\alpha$ de significación de .05.

\section{Resultados}

En cuanto a las medias de Atención, pudo observarse que los grupos de edad presentaron medias muy similares entre sí. Por su parte, en el caso de Claridad, el puntaje promedio de grupo de 15 a 24 años fue menor que el hallado en el grupo de 30 a 45 años y la media de éste, a su vez, menor que la hallada en el grupo de 60 a 75 años. En el caso de Reparación, el grupo de los más jóvenes presentó una media más baja que los dos grupos de mayor edad, y éstos últimos presentaron promedios similares entre sí.

A partir de la identificación de estas diferencias, se procedió a realizar un ANOVA. A partir de esta técnica estadística se pudo establecer que en la dimensión Atención Emocional los grupos no presentaron puntajes estadísticamente diferentes, mientras que en Claridad de Sentimientos y Reparación Emocional se encontraron diferencias significativas. En cuanto a Claridad, el grupo de 60 a 75 años presentó puntajes significativamente superiores a los del grupo de 15 a 24 (4.28; $\mathrm{p}<0.01)$ y del grupo de 30 a 45 (2.03; $\mathrm{p}<0.01)$. Respecto de Reparación, el grupo de 15 a 24 años presentó una media significativamente inferior a la del grupo $3(-3.50 ; \mathrm{p}<0.05)$ y al del grupo $2(-3.61$; $\mathrm{p}<0.01)$, mientras que éstos no se diferenciaron significativamente entre sí (ver Tabla 4).

Tabla 4 - Medias, desvíos y valores F de análisis de la varianza para Atención Emocional, Claridad de Sentimientos y Reparación Emocional según Grupo de edad.

\begin{tabular}{|c|c|c|c|c|c|c|}
\hline \multirow{2}{*}{ Grupo de edad } & \multicolumn{2}{|c|}{ Atención } & \multicolumn{2}{|c|}{ Claridad } & \multicolumn{2}{|c|}{ Reparación } \\
\hline & Media & Desvío & Media & Desvío & Media & Desvío \\
\hline 15 a 24 años & 24,34 & 7,01 & 25,11 & 5,72 & 26,04 & 6,43 \\
\hline 30 a 45 años & 24,34 & 6,70 & 27,36 & 6,35 & 29,66 & 6,18 \\
\hline 60 a 75 años & 23,96 & 7,07 & 29,40 & 6,25 & 29,54 & 6,25 \\
\hline Valor F & \multicolumn{2}{|c|}{0,08} & \multicolumn{2}{|c|}{$10,31^{\star \star}$} & \multicolumn{2}{|c|}{$8,51^{\text {** }}$} \\
\hline
\end{tabular}

${ }^{*} \mathrm{p}<0.05^{* *} p<0.01$ 
Los resultados generales brindan apoyo a la perspectiva positiva del envejecimiento, en tanto que el grupo de mayor edad presentó mejores puntuaciones en las habilidades de inteligencia emocional, lo que podría interpretarse como el logro de un mayor desarrollo en este aspecto. Específicamente, la capacidad de comprensión de las emociones de los adultos mayores fue mayor a la de los restantes grupos, y su habilidad de regulación fue superior a los más jóvenes y similar a la reportada por el grupo de 30 a 45 años. Estos resultados concuerdan con los antecedentes de investigación de Kafetsios (2004), Extremera, Fernández-Berrocal y Salovey (2006), Gross et al. (1997) y Nashiro, Sakaki y Mather (2011), entre otros.

En el presente estudio, la Atención emocional fue la única dimensión que no presentó características diferenciales en los grupos de edad. Kafetsios (2004), en una investigación realizada en Grecia, obtuvo resultados similares. Estos hallazgos aportan evidencia sobre el desarrollo temprano y la estabilidad de las habilidades de percepción y reconocimiento emocional a través de la vida, así como un desarrollo tardío de las capacidades de comprensión y regulación (LEPPÄNEN, 2011).

En cuanto a la Reparación Emocional, los adultos jóvenes y mayores superaron al grupo de 15 a 24 años respecto a la capacidad de modificar deliberadamente los estados emocionales. Esto implica que los dos grupos de mayor edad presentan más confianza en sus habilidades para modificar sus estados emocionales mediante estrategias reflexivas, sean sus emociones negativas o positivas. Asimismo, el grupo de adultos mayores superó a los grupos de menor edad en lo referido a Claridad de Sentimientos, es decir, que los adultos mayores de la muestra confían más en la eficacia de sus interpretaciones acerca de las dinámicas de su vida emocional y que disponen de mejores recursos lingüísticos para la comunicación de los afectos.

Siguiendo lo propuesto por Thayer et al. (2003) y Extremera y Fernández-Berrocal (2005), estos resultados pueden interpretarse en términos de perfiles. El grupo 1 presentó un perfil menos saludable de IEP, en tanto poseen similares niveles de atención emocional que los de más edad, pero menos claridad y reparación, lo que implicaría que poseen menores recursos para afrontar y procesar las vivencias emocionales. El grupo de 30 a 45 años, por su parte, presentó mejores habilidades que estos últimos en la comprensión y regulación de las emociones, por lo que presenta una configuración de inteligencia emocional más rica respecto al grupo de menor edad. Por último, el grupo de 60 a 75 años presenta mejores habilidades de comprensión emocional que los 
restantes grupos, dando cuenta de un procesamiento emocional más rico, con mayor capacidad de comprensión y expresión emocional e igualmente efectivo en lo relativo a la regulación emocional reactiva, ya que sus puntaciones en esta rama fueron similares. El conjunto de estos dos sets de habilidades hacen que los adultos mayores presenten mayores recursos y una configuración más rica y desarrollada de IEP que los restantes grupos de edad.

Tomando en cuenta los antecedentes empíricos respecto de Claridad de Sentimientos y de Reparación Emocional, es posible considerar diversas aristas de estos hallazgos. Los resultados de este estudio respecto de la Claridad de Sentimientos son coherentes con lo hallado en las investigaciones de Scheibe y Carstensen (2010), Blanchard-Fields (2007) y Extremera, Fernández-Berrocal y Salovey (2006). A su vez, contradicen la idea de que los adultos mayores presentan menor complejidad en su vida afectiva (LABOUVIE-VIEF et al., 2007). Como señalamos previamente, los procesos de regulación y comprensión emocional parecen estar modulados por diversos factores - edad (EXTREMERA; FERNÁNDEZ-BERROCAL; SALOVEY, 2006), socialización (MAUSS; BUNGE; GROSS, 2007), metas motivacionales (CARSTENSEN et al., 2011), centralidad del conflicto para el individuo (BLANCHARD-FIELDS; COATS, 2008), además de los factores cognitivos involucrados, por lo que consideramos que la contradicción con los hallazgos de Labouvie-Vief podría deberse a la complejidad misma del estudio de las emociones y a factores culturales, entre otros.

Trabajos previos señalan que el desempeño de los adultos mayores en problemáticas de corte emocional podría ser más eficaz en esa etapa de la vida debido al cambio en las metas motivacionales, ya que los adultos mayores se caracterizan por priorizar la obtención de bienestar emocional por sobre la información de otro tipo (CHARLES; CARSTENSEN, 2007). Asimismo, Blanchard-Fields (2007) afirma que en la vejez se produce una optimización del conocimiento situacional, estratégico y procedural de la regulación emocional debido a la experiencia vital (SCHEIBE; BLANCHARD-FIELDS, 2009). Estas dos perspectivas permiten pensar que los adultos mayores tengan mayores habilidades en la comprensión de sus vivencias emocionales gracias a que éstas se han vuelto más relevantes para su bienestar y porque a lo largo de la vida han acumulado experiencia y conocimiento en relación a su propia vida afectiva y a la de los demás.

En relación a la Reparación Emocional, los resultados señalan que las habilidades percibidas de la regulación de las emociones son menores en el grupo de 15 a 25 años en comparación con el grupo de 30 a 45 y el de 60 a 75 años, y estos dos grupos no se diferenciaron entre sí. Estos últimos resultados 
no concuerdan con la mayoría de los antecedentes, que señalan a los adultos mayores como el grupo etario con mayores y más complejas habilidades de Regulación de las emociones (BLANCHARD-FIELDS, 2007; KAFETSIOS, 2004; EXTREMERA; FERNÁNDEZ-BERROCAL; SALOVEY, 2006; GROSS et al., 1997). Dada la complejidad del fenómeno de la regulación de las emociones (KINKEAD BOUTÍN; GARRIDO ROJAS; URIBE ORTIZ, 2011) y de los numerosos aspectos que inciden en la eficacia de ésta, es probable que el instrumento utilizado no sea lo suficientemente sensible a las particularidades de la Reparación de las emociones en la vejez. Los estudios empíricos señalan que los adultos mayores prefieren controlar sus emociones mediante la selección de situaciones en las que se involucran (CHARLES; CARSTENSEN, 2007) y que presentan mayor flexibilidad que los adultos jóvenes en el afrontamiento de problemas poco estructurados, por lo que no siempre recurren a la regulación emocional reactiva o posterior al desarrollo de la emoción (SCHEIBE; CARSTENSEN, 2010; BLANCHARD-FIELDS, 2007), tal como evalúa la escala seleccionada para este estudio. Esto repercute en la necesidad de ajustar los instrumentos para lograr mayor validez al medir las características de la reparación de las emociones en la vejez.

En relación a la posibilidad de transferencia de este estudio, sus resultados podrían ser utilizados como insumos para el desarrollo de intervenciones específicas en prevención y promoción de la salud para el contexto cultural local.

Por ejemplo, sería posible diseñar dispositivos para el entrenamiento en estrategias de comprensión y regulación emocional para adolescentes y jóvenes, con el objetivo de fortalecer estas habilidades y así lograr un mayor ajuste emocional y bienestar psicológico. En cuanto a los adultos jóvenes, podrían diseñarse dispositivos similares a los ya mencionados, así como programas clínicos que hagan hincapié en el desarrollo de habilidades para profundizar la comprensión de las propias emociones y el aprendizaje de estrategias de regulación emocional, con el objetivo de facilitar la adaptación flexible a situaciones complejas y dinámicas. Respecto de los adultos mayores, resultaría de interés proyectar dispositivos orientados a la difusión de estos resultados para contrarrestar los efectos de los estereotipos sociales que afectan negativamente a este grupo etario, desempoderándolos (IACUB; ARIAS, 2011) en cuanto a su autonomía, comprensión y capacidad de adaptación a situaciones complejas y-o novedosas. La creación de dispositivos de reflexión y discusión que cuestionen los supuestos de deterioro cognitivo, depresión y enfermedades mentales (SÁNCHEZ PALACIOS, 2004) durante la vejez, así como la reflexión sobre sus propias habilidades de inteligencia 
emocional, podrían producir el incremento de eficacia de las mismas - ya que la creencia en la propia capacidad es un requisito para un buen desempeño - y impactar positivamente sobre las narrativas sobre la propia identidad de los mayores.

Si bien el presente estudio presenta alcances limitados y los resultados no pueden ser generalizados a la población, pueden elaborarse conclusiones del mismo así como delinear líneas futuras de investigación. Respecto de éstas últimas, resultaría importante, para lograr un mayor conocimiento del tema explorado, utilizar diversos tipos de instrumentos que permitan captar distintos aspectos que hacen a la complejidad del fenómeno estudiado. Por ejemplo, la utilización de cuestionarios y técnicas específicos para adultos mayores, así como la implementación de diseños de corte experimental y de triangulación metodológica, en los que se combinen estos tipos de abordajes con técnicas cualitativas.

En relación a los alcances, consideramos que las conclusiones de esta investigación dan cuenta de que las habilidades de Inteligencia Emocional, como parte del procesamiento emocional, se mantienen e incrementan su funcionamiento en la vejez. Debido a esto, pueden ser consideradas como un recurso eficaz y disponible para el logro de una adaptación exitosa frente a los desafíos de la vida cotidiana en esta etapa del desarrollo.

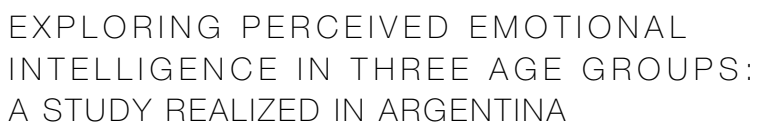

\section{abstract}

Perceived emotional intelligence is a set of abilities of emotional processing. It includes the capacity to recognize the own emotional states - Emotional attention -, the ability to understand and express them linguistically - Clarity of feelings - and also the ability to adaptively regulate them - Emotional repair. Developed within the Positive Psychology framework, it has been associated with resilience and works as a buffer for stress responses and also as a protective factor against the burnout. This study explores and compares the characteristics of perceived emotional intelligence in three age groups, 1) 15 to 24 ; 2) 30 to 45 and 3) 60 to 75 , using a cross-sectional, descriptive-correlational design. A non-probability intentional sample of 252 subjects, both male and female - for whom the Scale of Metacognition about Emotional States was administered - was 

than the other groups, and greater ability to regulate their emotions than the 15 to 24 group. This findings support previous studies which point out that the elderly have a more developed profile of perceived emotional intelligence abilities than younger people. This implies that the elderly have effective emotional resources to cope and adapt to everyday challenges.

keywords

Emotional Intelligence. Age Group. Positive Aging.

\author{
referencias
}

BLANCHARD-FIELDS, Fredda. Everyday problem solving and emotion - An adult developmental perspective. Current Directions in Psychological Science, Georgia, v. 16, n. 1, p. 26-31, Feb. 2007.

BLANCHARD-FIELDS, Fredda; COATS, Abby. The experience of anger and sadness in everyday problems impacts age differences in emotion regulation. Developmental Psychology, Washington, v. 44, n. 6, p. 1547-1556, Nov. 2008.

BLANCHARD-FIELDS, Fredda; STEIN, Renee; WATSON, Tonya. Age differences in emotion regulation strategies in handling everyday problems. Journals of Gerontology: Psychological Science, Oxford, v. 59, n. 6, p. 261-269, Nov. 2004.

BRIÑOL, Pablo; PEITY, Richard; RUCKER, Derek. The role of meta-cognitive processes in emotional intelligence. Psicothema, Oviedo, v.18, suppl., p. 26-33, Feb. 2006.

CARSTENSEN, Laura et al. Emotional experience improves with age: Evidence based on over 10 years of experience sampling. Psychology and Aging, Washington, v. 26, n. 1, p. 21-33, Mar. 2011.

CARSTENSEN, Laura; MIKELS, Joseph. At the intersection of emotion and cognition: Aging and the positivity effect. Current Directions in Psychological Science, Georgia, v.14, n. 3, p. 117-121, June 2005

CARSTENSEN, Laura; MIKELS, Joseph; MATHER, Mara. Aging and the intersection of cognition, motivation and emotion. In: BIRREN, James; SCHAIE, Warner (Org.). Handbook of the Psychology of Aging. San Diego: Academic Press, 2006. cap. 15. p. 343-362.

CHARLES, Susan; CARSTENSEN, Laura. Emotion regulation and aging. In: GROSS, James (Org.). Handbook of Emotion Regulation. New York: Guilford Press, 2007. cap. 15. p. 307-327.

DUVAL, Céline et al. Age effects on different components of theory of mind. Consciousness and Cognition, San Diego, v. 20, n. 3, p. 627-642, Sept. 2011.

EXTREMERA, Natalio; DURÁN, Auxiliadora; REY, Lourdes. Inteligencia emocional y su relación con los niveles de burnout, engagement y estrés en estudiantes universitarios. Revista de Educación, Madrid, n. 342, p. 239-256, enero/abr. 2007. 
EXTREMERA, Natalio; FERNÁNDEZ-BERROCAL, Pablo. Inteligencia emocional percibida y diferencias individuales en el meta-conocimiento de los estados emocionales: una revisión de los estudios con el TMMS. Ansiedad y Estrés, Madrid, v. 11, n. 2-3, p. 101-122, dic. 2005.

EXTREMERA, Natalio; FERNÁNDEZ-BERROCAL, Pablo; SALOVEY, Peter. Spanish version of the Mayer-Salovey-Caruso emotional intelligence test (MSCEIT). Version 2.0: reliabilities, age and gender differences. Psicothema, Oviedo, v. 18, suppl., p. 42-48, Oct. 2006

FERNÁNDEZ-BERROCAL, Pablo; EXTREMERA, Natalio; RAMOS, Natalia. Validity and reliability of the Spanish modified version of the Trait Meta-Mood Scale. Psychological Reports, Montana, v. 94, n. 3, p. 751-755, June 2004.

GROSS, James et al. Emotion and aging: Experience, expression, and control. Psychology and Aging, Washington, v. 12, n. 4, p. 590-599, Dec. 1997.

HAY, Elizabeth; DIEHL, Manfred. Emotion complexity and emotion regulation across adulthood. European Journal of Ageing, Amsterdam, v. 8, n. 3, p. 157-168, Sept. 2011.

IACUB, Ricardo; ARIAS, Claudia. Empoderamiento en la vejez. Journal of Behavior, Health and Social Issues, Del. Coyoacán, v. 2, n. 2, p. 25-32, nov. 2010 - abr. 2011.

KAFETSIOS, Konstantinos. Attachment and emotional intelligence abilities across the life course. Personality and Individual Differences, San Diego, v. 37, n. 1, p. 137-145, July 2004.

KINKEAD BOUTIN, Ana Patricia; GARRIDO ROJAS, Lusmenia; URIBE ORTIZ, Natalia. Modalidades Evaluativas en la Regulación Emocional: Aproximaciones actuales. Revista Argentina de Clínica Psicológica, Buenos Aires, v. 20, n. 1, p. 29-39, abr. 2011.

KOOLE, Sander; VAN DER LEEN, Lotte; SHEPPES, Gal. The Self-Regulation of Emotion. In: VOHS, Kathleen; BAUMEISTER, Roy (Org.). Handbook of Self-Regulation. New York: Guilford Press, 2011. cap 2. p. 22-40.

LABOUVIE-VIEF, Gisela et al. Six-year change in affect optimization and affect complexity across the adult life span: a further examination. Psychology and Aging, Washington, v. 22, n. 4, p. 738-751, Dec. 2007.

LAWTON, Powell et al. Dimensions of affective experience in three age groups. Psychology and Aging, Washington, v. 7, n. 2, p. 171-184, June 1992.

LEPPÄNEN, Jukka. Neural and Developmental Bases of the Ability to Recognize Social Signals of Emotions. Emotion Review, San Diego, v. 3, n. 2, p. 179-188, Apr. 2011.

LOBUE, Vanessa; DE LOACHE, Judy. Superior detection of threat-relevant stimuli in infancy. Developmental Science, Singapore, v.13, n. 1, p. 221-228, Jan. 2010.

MÁRQUEZ GONZÁLEZ, María et al. Experiencia y Regulación emocional a lo largo de etapa adulta del ciclo vital: análisis comparativo en tres grupos de edad. Psicothema, Oviedo, v. 20, n. 4, p. 616-622, nov. 2008.

MAUSS, Iris; BUNGE, Silvia; GROSS, James. Automatic emotion regulation. Social and Personality Psychology Compass, San Francisco, v. 1, n. 1, p. 146-167, Nov. 2007.

MAYER, John; CARUSO, David; SALOVEY, Peter. Models of Emotional Intelligence. In: STERNBERG, Robert (Org.). Handbook of intelligence. Cambridge: Cambridge University Press, 2000. cap. 18. p. 396-420.

MAYER, John; SALOVEY, Peter. What is Emotional Intelligence? In: SALOVEY, Peter; SLUYTER, David (Org.). Emotional Development and Emotional Intelligence: Educational Implications. New York: Harper Collins, 1997. cap 1. p. 3-34. 
MAYER, John; STEVENS, Alexander. An emerging understanding of the reflective (meta-) experience of mood. Journal of Research in Personality, San Diego, v. 28, n. 3, p. 351-373, Sept. 1994.

MENDES, Wendy Berry. Weakened Links Between Mind and Body in Older Age: The Case for Maturational Dualism in the Experience of Emotion. Emotion Review, San Diego, v. 2, n. 3, p. 240-244, July 2010.

NASHIRO, Kaoru; SAKAKI, Michiko; MATHER, Mara. Age differences in brain activity during emotion processing: Reflections of age-related decline or increased emotion regulation? Gerontology, Innsbruck, v. 58, n. 2, p. 156-163, Feb. 2011.

PASCALIS, Olivier et al. Plasticity of face processing in infancy. Proceedings of the National Academy of Sciences of the United States of America, Washington, v. 102, n. 14, p. 5297-5300, Apr. 2005.

RAMOS-DÍAZ, Natalia; FERNÁNDEZ-BERROCAL, Pablo; EXTREMERA, Natalio. Perceived emotional intelligence facilitates Cognitive-Emotional Processes of Adaptation to an Acute Stressor. Cognition \& Emotion, London, v. 21, n. 4, p. 758-772, May 2007.

REGNER, Evangelina. Estudio de las propiedades psicométricas de la Escala de Metaconocimiento de Estados Emocionales (TMMS-24) en Argentina. In: ENCUENTRO IBEROAMERICANO DE PSICOLOGÍA POSITIVA: la psicología positiva y sus aplicaciones, 4., 2009, Buenos Aires. Anais... Buenos Aires: Universidad de Palermo, 2009. p. 22.

SALGUERO, José et al. Measuring perceived emotional intelligence in the adolescent population: Psychometric properties of the Trait Meta-Mood Scale. Social Behavior and Personality, Palmerston North, v. 38, n. 9, p. 1197-1210, Oct. 2010.

SÁNCHEZ PALACIOS, Concepción. Estereotipos negativos hacia la vejez y su relación con variables sociodemográficas, psicosociales y psicológicas. 2004. 281 p. Tese (Doctorado en Psicología Evolutiva y Educación) - Departamento de Psicología Evolutiva y de la Educación, Universidad de Málaga, Málaga, 2004.

SCHEIBE, Susan; CARSTENSEN, Laura. Emotional aging: Recent findings and future trends. Journal of Gerontology: Psychological Science, Oxford, v. 65B, n. 2, p. 135-144, Mar. 2010

SCHEIBE, Susanne; BLANCHARD-FIELDS, Fredda. Effects of Regulating Emotions on Cognitive Performance: What is Costly for Young Adults Is not so Costly for Older Adults. Psychology and Aging, Washington, v. 24, n. 1, p. 217-223, Mar. 2009.

SHIOTA, Michelle; LEVENSON, Robert. Effects of aging on experimentally instructed detached reappraisal, positive reappraisal, and emotional behavior suppression. Psychology and Aging, Washington, v. 24, n. 4, p. 890-900, Dec. 2009.

THAYER, Julian et al. Gender Differences in the Relationship between Emotional Regulation and Depressive Symptoms. Cognitive therapy and research, Boston, v. 27, n. 3, p. 349-364, June 2003

Recebido: 09/08/2012

Aceite Final: 06/02/2014 